\title{
AU SUJET D'ACCIDENTS CONSECUTIFS AUX INJECTIONS DE BAYER 205 CHEZ DES ÉTALONS DOURINÉS
}

\author{
Par H. VELU, J. BAROTTE, L. BALOZET et G. LAVIER
}

Au cours de nos essais sur les propriétés thérapeutiques du Bayer 205 dans les trypanosomoses animales au Maroc, nous avons eu l'occasion de traiter par ce produit, ensemble et parallèlement, de fin juin à fin décembre 1923 , quatre étalons et un baudet atteints cliniquement de dourine.

Laissant de côté les résultats chimiothérapeutiques obtenus qui ont fait l'objet d'une note précédente, il nous a paru intéressant de signaler les accidents consécutifs aux injections intraveineuses de Bayer 205 que nous avons observés au cours du traitement.

Deux étalons (Mylord et BeKaI) ont reçu sans manifester de réaction appréciable en août et novembre ( 7 et 9 août-11 et 14 novembre) la dose de 7 grammes du produit, en deux injections intraveineuses : la première, de 4 grammes pour tâter la sensibilité du malade, la deuxième (le lendemain ou le surlendemain) de 3 grammes pour compléter la dose totale.

Deux autres étalons (Mesquin et Frigi) et un baudet (Diabolo), traités dans les mêmes conditions, les 17 et 18 aoùt, puis les 11 et 14 novembre, présentèrent des accidents graves dont un mortel (Mesquin), aux premières injections, en août ; les survivants supportèrent, par contre, sans aucune réaction, les deuxièmes injections, en novembre.

Evolution des accidents. - La description clinique complète des accidents, pour chaque malade, dépasserait par sa longueur le cadre de cette simple note. De gravité variable pour chacun des trois sujets, dont un seul est mort, la similitude des symptômes nous permettra de les grouper en un résumé synthétisant les caractères les plus typiques de ces accidents.

Dans le cours de l'après-midi qui suit immédiatement l'injection, un léger abattement général, sans température, auquel correspond une petite diminution d'appétit, décèle à peine un changement dans l'état normal du sujet, état qui passerait vraisemblablement inaperçu si l'infirmier n'était prévenu de surveiller plus particulièrement ces malades.

Le lendemain matin, quelques œdèmes caractéristiques de dou-

Annales de Parasitologie, T. III, $\mathrm{N}^{\circ}$ 1. - Janvier 1925, p. 12-20. 
rine, en plaques arrondies, réapparaissent plus ou moins nombreux ou, s'ils existaient la veille, s'accusent dans leurs dimensions par leur saillie plus forte. Un peu d'œdème diffus plus ou moins prononcé des organes génitaux (verge, fourreau ou bourses), quelques empâtements ganglionnaires dans l'auge complètent éventuellement le tableau de la légère réaction que nous avons notée chez presque tous les chevaux recevant leur première dose de 4 grammes de 205 . Aucune aggravation ne marque l'injection de la dose complémentaire de 3 grammes, le lendemain ou le surlendemain, et en 48 heures, plaques et œdèmes disparus, l'animal a repris toute sa vivacité et ses allures, avec toutes les apparences de la santé. C'est là le tableau normal.

Pour les animaux chez lesquels nous avons observé des accidents, ceux-ci s'annoncent, dès le lendemain matin, par l'accentuation des réactions œedémateuses.

L'étalon qui est mort par la suite présentait, sur toute la surface du corps, de petites boursouflures œdémateuses, rondes ou ovales, du volume d'une noix à celui d'un œuf, rappelant par leur aspect d'ensemble, quoique moins durés au toucher, certains cas déjà observés d'une multitude de petites tumeurs sarcomateuses généralisées.

L'auge est complètement empâtée avec des ganglions fortement hypertrophiés et œedémateux ; on note de forts œdèmes de la région parotidienne envahissant un peu les régions voisines de l'encolure, de l'œdème de la face et particulièrement du chanfrein, des nasaux et des lèvres rappelant l'anasarque.

Chez les sujets moins gravement atteints, mêmes symptômes mais atténués :

Plaques de dourine très accentuées.

Petites boursouflures œéémateuses sous-cutanées faisant songer, par régions, à une forte poussée d'échauboulure.

Empâtement de l'auge, avec petite réaction ganglionnaire et de la région parotidienne.

Quelques œdèmes des lèvres, même de la joue, au passage du licol et des organes génitaux externes.

En 36 à 48 heures, ces œdèmes rétrocèdent faisant place à de véritables érythèmes. Toutes les parties glabres ou à poils fins prennent une teinte rosée très visible même à travers les poils.

Le bout du nez, les commissures des lèvres sont par endroits rougeâtres ou violacés, sensibles, l'animal accuse de violentes démangeaisons, de même qu'à l'ars ou au pli du grasset, au voisinage du fourreau, en un mot, partout où la peau, fine, prend les mêmes colorations. 
A partir de ce moment le malade, littéralement prostré, cesse de manger et ne sort de son abattement que pour essayer de se gratter ou de se mordre le paturon et la couronne. Ces régions sont également vivement colorées, érythémateuses, parfois même avec de petites éruptions papuleuses ou vésiculeuses, particulièrement au niveau du bourrelet qui est chaud, gonflé, douloureux, suintant par endroits et paraît le siège d'un prurit particulièrement intolérable ; les parois du sabot par leur chaleur et leur sensibilité à la percussion rappellent la fourbure.

Aucune réaction thermique caractéristique n'a été relevée au cours de l'évolution des accidents ; la température fit dans les derniers jours quelques ascensions irrégulières, dont aucune ne dépassa $39^{\circ}$.

Dans un état de prostration complète, les yeux mi-clos, cherchant l'obscurité, le malade laisse souvent pendre partiellement la langue, les lèvres inertes, appuyant l'extrémité inférieure de la tête ou les arcades incisives sur le rebord de la mangeoire en guise de point d'appui. Il refuse toute nourriture et maigrit avec une rapidité effrayante accusant plutôt de la polyurie. En huit jours, l'étalon était arrivé à un état littéralement squelettique. Bien entendu, tout déplacement du sujet devient très vite impossible, celui-ci vacillant au moindre mouvement, très difficile à relever lorsqu'il a essayé de se coucher.

Nous avons noté également une tendance très nette aux eschares rapides ; dès que l'animal se couche, de larges eschares transforment en peu de temps toutes les parties saillantes : l'orbite, épaule, hanches, boulet, jarret, en larges plaies.

C'est dans cet état que meurt, 11 jours après la deuxième injection, l'étalon Mesquin.

Lésions d'autopsie. - Outre les lésions externes signalées avant la mort du malade : érythèmes, éruptions, amaigrissement intense, larges eschares, le cadavre très émacié rappelle par la couleur de ses masses musculaires l'aspect et la coloration caractéristiques des viandes fiévreuses, à muscles cuits et friables.

Le poumon est normal, le foie légèrement augmenté de, volume, jaunâtre, décoloré, se rupturant facilement ; tous les autres organes n'accusent qu'une forte congestion et présentent sur leur coupe des vaisseaux très largement dilatés, gorgés de sang imparfaitement coagulé.

Le caractère typique qui frappe réellement le praticien à l'ouverture du cadavre est la constatation, partout, dans tous les organes, dans toutes les régions, de phénomènes de vaso-dilatation intense. Dans le conjonctif, sur les séreuses pariétales ou viscérales (sans 
grands épanchements d'ailleurs), à la surface des intestins, dans le mésentère, etc..., on note de fortes et larges arborisations vasculaires, les moindres petits vaisseaux paraissent dilatés à l'extrême ainsi qu'on les retrouve sur la coupe des organes.

Cette vaso-dilatation est plus particulièrement intense encore au niveau des organes génitaux où de petites veinules gorgées, moniliformes atteignent la taille d'un crayon ou du petit doigt, accusant une disproportion plus flagrante encore que partout ailleurs entre leur volume et le territoire irrigué. Même constatation pour quelques ganglions médiastinaux, les organes génito-urinaires et de leurs annexes.

Traitement. - Croyant primitivement à des accidents d'origine toxique et en raison de la voie particulière d'élimination du médicament par les urines notre action thérapeutique s'était bornée en dehors des soins élémentaires d'hygiène et d'alimentation, à activer la diurèse et l'élimination intestinale (bicarbonate de soude, purgatifs légers, pilocarpine à très petites doses) en soutenant le cœur (injections de caféine, "d'ailleurs mal tolérées, en injections souscutanées et le plus souvent éliminées avec de larges eschares).

La constatation de phénomènes de vaso-dilatation générale aussi exceptionnellement intenses à l'autopsie du premier malade nous incita à utiliser, sans délai, sur l'autre étalon et le baudet des injections d'adrénaline en solution acide à 1 p. 1000 .

Le soir même, $12 \mathrm{cc}$. de la solution étaient injectés par voie hýpodermique à chaque malade; le lendemain ceux-ci recevaient $10 \mathrm{cc}$. le matin et $10 \mathrm{cc}$. le soir complétés par une troisième injection de $10 \mathrm{cc}$. le troisième jour.

Bien que l'état des deux malades fût grave, une notable amélioration se manifesta dès les premières injections d'adrénaline qui

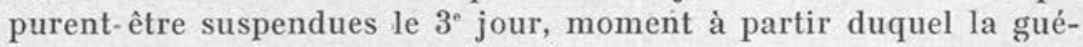
rison ne fit que s'affirmer.

Accidents particuliers. - Sur l'étalon ayant guéri, nous avons pu constater l'évolution lente d'une lésion particulièrement curieuse. Quelques jours après le début des premiers symptômes apparaissaient sur la croupe deux plaques œémateuses partant de la soudure iléo-sacrée et s'étalant en éventail sur la croupe, de part et d'autre, dans une situation très exactement symétrique par rapport à la ligne médiane (fig.).

Pendant une huitaine de jours, la lésion se présentait - le matin, au réveil - sous l'aspect d'une plaque d'œdème à saillie très accusée, assez dure, bien que conservant à la pression l'ênpreinte du doigt, légèrement humide sans être suintante, le poil 
hérissé à sa surface marquant encore plus nettement le contraste avec les régions voisines normales.

$\mathrm{Au}$ cours de la journée, la lésion s'aplatissait progressivement tout en conservant sa forme jusqu'à se présenter - le soir - en dépression par rapport aux parties voisines et comme couverte d'un tégument dur, épaissi, raccorni, donnant l'impression d'une. eschare sèche, prête à s'éliminer.

Le lendemain matin, la lésion toujours identique dans sa forme et strictement symétrique par rapport à la ligne médiane repre-. nait son aspect œdémateux.

Très nettes les premiers jours, ces variations d'aspect s'atté-
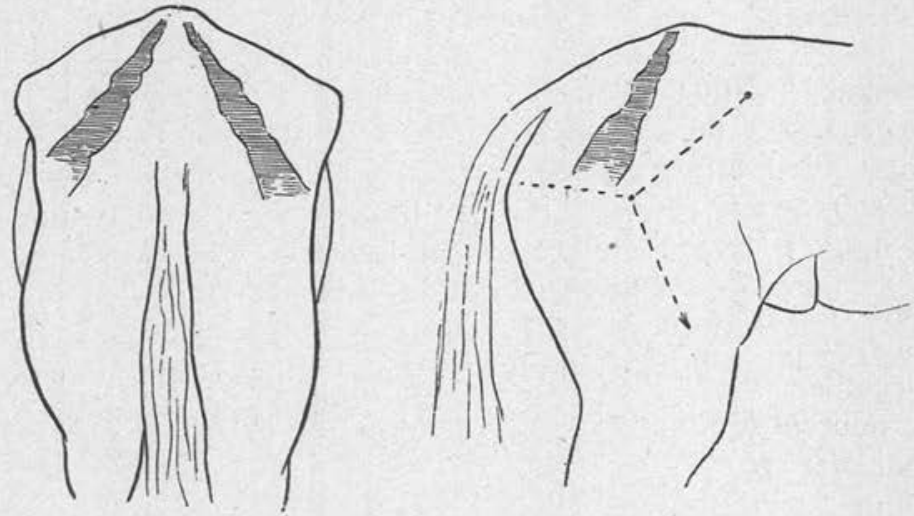

- Fig. - Lésions œdémateuses de la croupe de l'étalon Frigi.

nuaient progressivement ; seule, la lésion, avec sa forme si caractéristique, persistait longuement; aucune eschare ne s'étant formée, la surface se couvrit de petites vésicules croûteuses entraî. nant en s'éliminant une grande partie des poils.

Au début de janvier 1924, la trace de la lésion était encore très visible, marquée par une desquamation abondante de l'épiderme, un furfur épidermique blanchâtre, accusé encore par la rareté des poils et par le fait que quelques-uns repoussaient en blanc.

Même constatation était d'ailleurs faite au niveau de diverses parties fines (pli du coude, pli du grasset, pli du flanc) où après la disparition des érythèmes très nets du début et la chute d'une partie des poils ceux-ci repoussèrent blancs ou gris formant ainsi sur le fond de la robe de l'animal' une série de " taches accidentelles " à siège très anormal pour qui n'avait pas suivi l'évolution des accidents.

Cette localisation très particulière à unè zone nettement symé- 
trique par rapport à la ligne médiane, affectant certainement un même territoire vasculo-nerveux bien délimité, siège d'une lésion à évolution lente semble caractéristique de troubles trophiques vasculo-nerveux à rapprocher de la tendance très nette aux eschares présentées par les malades.

Recherches sur la pathologie des accidents. - Ces accidents survenant simultanément sur trois malades, $\mathbf{1 0}$ jours après l'applieation sans incident d'un traitement identique sur deux autres étalons dourinés, ne laissaient pas que d'être quelque peu surprenants bien qu'ils aient déjà été signalés dans l'emploi du 205 Bayer.

Il était naturel d'en éliminer suecessivement les causes possibles.

Posologie. - Nous avons pris comme base de la posologie chez le cheval la dose de 7 gr. généralement acceptée.

Balozet avait utilisé le médicament à ce taux en trois injections ( 1,4 et deux grammes) sans le moindre accident en septembre 1922.

Nos deux premiers malades traités les 7 et 9 août n'avaient d'ailleurs marqué à deux injections successives de 4 et 3 grammes aucune réaction inquiétante.

Le coefficient chimiothérapeutique de 167 (Mayer et Zeiss) admis pour le Bayer 205 éliminait, par ailleurs, toute hypothèse d'intoxication par un voisinage possible des doses thérapeutiques et toxiques.

Conservation du produit. Un concours de circonstances fortuites n'ayant pas permis l'emploi de la solution à 10 p. 100 du 205 dans de l'eau distillée stérile, immédiatement après sa préparation et cette solution n'ayant été injectée que 8 jours plus tard la conservation du produit pouvait être suspectée.

La solution a été laissée en ballon stérile à l'obscurité, dans la glacière ; aucune altération macroscopique n'avait été relevée au moment même de l'injection.

Notons également en faveur de la longue conservation du produit le fait que nos deux premiers malades avaient reçu sans inconvénient des doses de 7 grammes de 205 constituées en grande partie de solution en ampoules préparées au moment des premiers essais de Balozet, en septembre 1922, soit depuis environ 10 mois.

Toxicité spéciale d'un échantillon du produit. - Le 205, cause des accidents, provenant d'un flacon récemment entamé, on pourrait envisager l'hypothèse soit d'une altération possible du produit en poudre, soit d'une toxicité particulière à cet échantillon analogue aux "séries toxiques " signalées pour le novarsenobenzol (fait 
d'ailleurs peu probable en raison du peu de toxicité normale du médicament).

Dans le but d'éliminer ces dernières hypothèses, deux inoculations de contrôle furent pratiquées à des chiens.

Un animal étant inoculé par voie intraveineuse avec 10 centigrammes du produit incriminé en solution à 10 p. 100 employée dès sa préparation.

L'autre recevant 10 jours plus tard une dose identique de cette mème solution conservée en ballon stérile dans les conditions de conservation suspectées précédemment pour les chevaux.

Aucun de ces animaux ne présenta la moindre réaction ni le moindre trouble consécutif.

Rapprochement avec les accidents du néo-salvarsan dans le traitement de la syphilis. - Sans parler prématurément d'analogie entre les accidents que nous venons de décrire et ceux dus au néosalvarsan dans le traitement de la syphilis, on ne peut s'empêcher d'établir entre eux un rapprochement tant au point de vue symptomatologique que dans les hypothèses sur lesquelles peuvent s'étayer leur pathogénie.

Parmi ces accidents " on a signalé l'urticaire, l'herpès, le zona, des érythèmes morbiliformes, scarlatiniformes parfois un œè̀me passager de la face et des extrémités " (Leredde).

Ce sont là d'ailleurs avec les névrites arsenicales et la fièvre, les moindres accidents du néo-salvarsan, les plus graves comportant des paraplégies et même des cas mortels.

Au nombre des accidents arséno-benzoliques, il faut mentionner " les arsenobenzolides, éruptions cutanées pouvant apparaître soit précocement, soit tardivement et allant depuis le simple érythème jusqu'au pemphigus foliacé en passant par l'urticaire, l'érythème polymorphe, les dermatites vésiculeuses et bulleuses et aussi les troubles vaso-constrictifs en opposition avec les vaso-dilatateurs plus habituels et provoquant un syndrome de Raynaud passager ou durable, avec ou sans gangrène des extrémités, symétrique ou non " (Nicolas, Massia et Dupasquier).

Dans la prophylaxie de ces accidents une grande importance s'attache à l'emploi pour la solution d'eau distillée, stérilisée après distillation et à l'emploi immédiat de la solution ainsi préparée.

Le rapprochement des injections (technique de Schreiber) aurait donné avec le néo-salvarsan plus d'accidents que l'élévation des doses du médicament.

Dans les cas particuliers qui nous intéressent, on peut se demander si le rapprochement, à 48 heures ou trois jours d'intervalle, des deux doses complémentaires de 4 et 3 grammes de 205 
n'est pas à incriminer. Le fractionnement de la dose totale de 7 grammes avait pour but de tâter la sensibilité individuelle du malade au médicament; or, il est possible, en se basant sur les données acquises pour le néo-salvarsan de se demander si le cheval ne supporterait pas mieux la dose totale d'emblée.

Sensibilité individuelle, idiosyncrasie, intolérance individuelle. - Ces facteurs étiologiques paraisśent peu acceptables pour des accidents observés sur trois sujets injectés le même jour, quand deux injectés précédemment n'avaient marqué aucune réaction.

D'ailleurs voici ce qu'écrit Leredde dans son étude des accidents du salvarsan: "Les fautes de technique éliminées, il faut chercher l'origine des petits accidents, comme des accidents graves, dans l'état du malade antérieur à l'injection " .

"Dans d'autres cas, l'action toxique ne s'explique plus bien je fais allusion à ceux dans lesquels on observe des érythèmes ce sont les cas où tout le monde parle d'idiosyncrasie, d'intolérance individuelle, qu'il s'agisse de salvarsan, d'iodure de potassium, de mercure, d'opium, de chloral, etc... Quelques auteurs rapprochent le mécanisme des éruptions médicamenteuses de celui de l'anaphylaxie. Les théories que j'ai exposées sur les hématodermites me conduisent également à rechercher dans un état du milieu sanguin antérieur à l'absorption d'un agent chimique, l'origine d'accidents qui ne se produisent pas chez la très grande majorité des malades, des intoxications qu'on explique par l'" idiosyncrasie ", en attendant le jour où on n'osera plus se servir d'un mot qui indique notre ignorance, et surtout le jour où le mécanisme précis sera déterminé ".

Un autre fait à lui seul écarterait toute hypothèse d'intolérance individuelle, c'est l'innocuité absolue du traitement renouvelé, aux mêmes doses, chez les deux malades survivants les 11 et 14 novembre, qui ne présentèrent pas la moindre réaction aux deuxièmes injections.

Réaction de Herxheimer. - Dans son même travail, Leređde se demande logiquement si, à côté des conditions individuelles en raison desquelles un agent thérapeutique, non toxique normalement aux doses habituelles couramment employées devient sensiblement toxique, il n'y a pas d'autres causes à incriminer?

"Certes on ne doit pas tout expliquer par la réaction de Herxheimer ; il n'est pas douteux toutefois qu'un grand nombre de symptômes sont dus à la destruction des spirochètes, aux phénomènes de congestion et d'œdème qui en sont la conséquence. La réactivation biologique (Gennerich, Milian) qui, remarquons-le en pas- 
sant, peut survenir, einq, dix, quinze, vingt jours après une injection, rentre dans le cadre de la réaction de Herxheimer ".

Celle-ci peut se produire plus ou moins richement au niveau de tout foyer syphilitique. "Les accidents qui la traduisent sont le plus souvent bénins, spontanément et rapidement curables, mais on conçoit qu'elle puisse déterminer, lorsqu'elle se produit au niveau d'un foyer méningé ou encéphalique, des accidents très graves entraînant souvent la mort ".

Cette réaction est due à l'efficacité même de l'agent anti-syphilitique. $\mathrm{Si}$, avec le mercure et l'iodure on l'observe plus rarement qu'avec le salvarsan, e'est que ces médicaments ont une action moindre sur les lésions syphilitiques. A mesure que nous découvrirons des agents anti-syphilitiques plus actifs, ees réactions se produiront de plus en plus fréquentes et le médecin devra savoir, pour chaque malade, régler les doses de façon à éviter les réactions violentes et à prévenir les accidents, tout en agissant sur l'infecticin d'une façon énergique.

Bien que des réserves aient déjà été faites au sujet de l'applieation thérapeutique absolue du Bayer 205 chez les chevaux infectés de dourine dans les conditions cliniques normales, il est hors de doute que le produit jouit de propriétés trypanocides très accusées vérifiées expérimentalement. C'est dans cette action trypanocide violente, réagissant sur un organisme infecté, dans la destruction de nombreux trypanosomes et dans les phénomènes de congestion et d'œdème qui en sont la conséquence que nous aurions tendance à voir les facteurs étiologiques principaux des accidents que nous avons observés.

Il ne faut d'ailleurs pas en déduire que l'on doive considérer comme secondaires les précautions très rigoureuses de technique dans l'emploi du produit, en première ligne desquelles nous placerons :

La préparation extemporanée des solutions avec de l'eau distillée, stérilisée après distillation.

L'emploi immédiat de ces solutions.

En cas d'impossibilité d'emploi immédiat, la mise aussi rapide que possible en ampoules bien pleines, scellées et stérilisées à nouveau.

Nous laisserons encore à de plús complètes expérimentations, le soin de préciser l'opportunité de fractionner en deux injections rapprochées la dose thérapeutique globale à administrer à un malade ou de l'utiliser d'emblée.

Travail du Laboratoire de Recherches du Service de lElevage, Casablanca. 